\title{
Seroprevalence and Risk Factors Associated with Measles outbreaks among Children in Kwale, Lamu and Narok Counties in Kenya, 20I4
}

\begin{abstract}
Background: Measles burden has been on the decline globally since 1980 due to universal use of measles vaccine; with outbreaks reported in Africa, Europe and Asia (2008). Population immunity assessment is key to determining progress towards elimination of measles as advised by World Health Organisation (WHO).

Objectives: This study aimed to determine the sero-prevalence and risk factors associated with measles outbreaks among children aged 9 to 59 months in Kwale, Narok and Lamu Counties of Kenya.

Methods: This was a hospital-based cross-sectional descriptive study in which 453 children were studied. Very ill children were excluded. A structured questionnaire was used to capture participants' demographics and risk factors. Approximately $50 \mu 1$ of blood were collected, dried blood spots (DBS) prepared and transported to KEMRI laboratory for analyses. DBS were used for detection of measles IgG antibodies using the NOVATEC Immunodiagnostic GMBH (Nova Lisa ${ }^{\mathrm{TM}}$ ELISA) (Germany). Serology results were correlated with actual vaccination coverage, demographic and risk factors using statistical package (SPSS) version 20. Numbers and percentages of positive, negative and equivocal sera were found, Chi-square used to compare proportions and a $95 \%$ confidence interval used to describe limits of percentages. A p-value of less than 0.05 was considered statistically significant.
\end{abstract}

Results: A total of 453 children were recruited, $233(51.4 \%)$ male and $220(48.6 \%)$ female $413 / 453(91.2 \%)$ of guardians were aware about measles vaccines, $40(8.8 \%)$ were not $(\mathrm{P}<0.001)$. 280/413 \{(67.8\%) $95 \% \mathrm{CI} ; 61.3 \%$ to $74.3 \%)\}$ received information from health workers while $133 / 413\{(32.2 \%) 95 \%$ CI; $29.1 \%$ to $35.3 \%)\}$ got information from elsewhere $(\mathrm{P}<0.001)$. 408/453 $\{(90.1 \%) 95 \% \mathrm{CI} ; 81.8 \%$ to $98.4 \%)\}$ were vaccinated, while only $11 / 453\{(2.4 \%) 95 \% \mathrm{CI} ; 2.2 \%$ to $2.6 \%)\}$ were not vaccinated. $34 / 453\{(7.5 \%) 95 \% \mathrm{CI}$; $6.9 \%$ to $8.1 \%)$ \} could not ascertain their vaccination status. 346/453(76.4\%) children had vaccination cards, $107(23.6 \%)$ did not $(\mathrm{P}<0.001)$. Overall, 376/453 \{(83.0\%) 95\% CI; $75.4 \%$ to $90.6 \%)\}$ children had protective antibody titres, while $77 / 453\{(17.0 \%) 95 \% \mathrm{CI}$; $15.5 \%$ to $18.7 \%)$ \} did not $(\mathrm{P}<0.001)$.

Conclusion: A huge number of children did not have protective antibody titres against measles despite the wide vaccine coverage. Vaccination awareness was low with large number of children lacking records. Large scale assessment of measles population immunity and measles vaccine awareness is required in Kenya.

Keywords: Sero-prevalence; Sero-protectively; Herd immunity; Risk factors; Measles; Vaccines
Volume 5 Issue 5 - 2017

\author{
Ali Juma Kanga,' Serah Kaggia, ${ }^{2}$ Juliette R \\ Ongus, ${ }^{3}$ Raphael M Lwembe ${ }^{4}$ \\ 'Medical Sciences Department, Technical University of Mombasa, \\ Kenya \\ ${ }^{2} \mathrm{~S}$ chool of medicine, Jomo Kenyatta University of Agriculture \\ and Technology, Kenya \\ ${ }^{3}$ Medical Laboratory Sciences department, Jomo Kenyatta \\ University of Agriculture and Technology, Kenya \\ ${ }^{4}$ Centre for Virus Research, Kenya Medical Research Institute, \\ Kenya
}

Correspondence: Ali Juma Kanga, Technical University of Mombasa, P.O. Box 90420-80100, Mombasa, Kenya, Tel 25441 2492222/3; +254 072I-545650, Fax 254 4I-2495632; Email alikanga@tum.ac.ke; akanga74@gmail.com

Received: June 27, 2017 | Published: July 19, 2017

\section{Abbreviations}

$\mu 1$ : Micro Litre; CAR: Central Africa Republic; CI: Confidence Interval; DBS: Dry Blood Spot; ELISA: Enzyme Linked Immunosorbent Assay; IgG: Immunoglobulin Gamma; KEMRI: Kenya Medical Research Institute; McV: Measles Containing Vaccine; mIU: Mill International Unit; Ml: Milli litre; NTU: Nova Tec Units; OR: Odds Ratio; SPSS: Statistical Package for Social Sciences; WHO: World Health Organization

\section{Introduction}

Measles is an acute, highly contagious viral disease estimated to cause over 158,000 annual deaths globally by $2011 .^{1-3}$ Measles is highly communicable, with greater than $90 \%$ secondary attack rates among susceptible persons. ${ }^{4,5}$ In the pre vaccination period, Measles used to be a universal childhood disease with attack rates that went beyond $90 \%$. Consequently, in the 10th century a Persian physician, Rhazes (Abu Bakr Muhammad Ibn Zakariyya al-Razi), described it as a viral disease more dreadful than small pox. ${ }^{6,7}$ The mortality and morbidity of measles however substantially decreased with the discovery of measles vaccine in the early $1960 \mathrm{~s}^{3}{ }^{3}$ Nevertheless, Measles virus still remains an important cause of vaccine-preventable disease, disability and death among children worldwide, especially in resource-poor countries. In the year 2001, Measles accounted for a loss of over 23 million disability-adjusted life years globally. ${ }^{4}$ Elsewhere, between 2008 and 2011, France surprisingly experienced a series of outbreaks with more than 20,000 cases at a time they were expecting to have been achieving measles elimination. ${ }^{8}$ Kenya experienced a nationwide Measles outbreak from December 2010 to 2012. ${ }^{9,10}$ Despite the reported high measles immunisation coverage of $85 \%-90 \%$ that followed, in 2012 , repeat outbreaks occurred that comprised 3,056 cases of which 767 were confirmed, an increase of 102 confirmed cases from 2011 outbreaks. ${ }^{9} 10$ This prompted a massive immunisation programme that covered more than $99 \%$ of the target population (9 - 59 months old children). ${ }^{11-13}$ Genetic characterisation of measles virus has revealed 24 genotypes distributed within 8 
genotypic groups as; A, B1-3, C1-2, D1-11, E, F, G1-3, and H1-2. ${ }^{14}$ Phenotypically, measles virus is mono topic; hence we have only one serotype..$^{15}$ Thus, immunity from one genotypically distinct measles virus will essentially protect against all other genotypes with no significant difference in sero-conversion rates. ${ }^{16,17}$ Live attenuated measles virus vaccine is stable and can prevent measles effectively. ${ }^{16,18}$ There is a range of attenuated measles vaccines available, used singly or in combination as measles containing vaccines $(\mathrm{McV})$ with either mumps, Rubella or Varicella. ${ }^{19}$ Vaccines currently in use mostly come from the Edmonston lineage strains such as Schwarz, AIK-C, Zagreb, Rubeovax, and Moraten. ${ }^{19,20}$ A few vaccines are made from wild measles virus isolates; CAM-70 from Japan, Shanghai-191 and Changchun-47 from China, and Leningrad-4 from Russia. ${ }^{17,19}$ ${ }^{21}$ Edmonton - Zagreb vaccine strain was used in our study, the mostly used measles vaccine strain in Kenya and most other low economy countries and is effective against the circulating measles genotypes.. ${ }^{18,22,23}$ Edmonton - Zagreb vaccine strain has shown proven efficacy when used at 9 months down to 4.5 months of recipient ages and seems to work well even in the face of existing maternal antibodies..$^{22,23}$ Among the key indicators of protection against Measles and by extension a pointer of Measles elimination, is adequate serological evidence of Measles immunity. ${ }^{24-26}$ Records on population immunity are an excellent pointer of the progress towards measles elimination, this data can be got from well-conducted sero-prevalence studies. ${ }^{27,28}$ In Kenya, sero-surveillance of neutralising antibodies against measles is not routinely conducted; instead, incidence reports are largely relied upon. Therefore, it is difficult to determine whether adequate levels of immunity exist in the country. The primary purpose of this study was to conduct a sero-survey to determine measles immune globulin levels among a sub-population of children aged 9 to 59 months attending selected health facilities in Kwale, Lamu and Narok Counties. Secondarily, we looked at the risk factors associated with low measles sero-protectively among children in the 3 Kenyan counties that experienced measles outbreak in varying degrees in previous outbreaks. Would be nice to add a paragraph on situation analyses of reported risk factors.

\section{Methods}

\section{Study sites}

The study was conducted in hospitals in three counties Kwale, Lamu and Narok of the republic of Kenya which experienced measles outbreak in the year.

Kwale County: Part of the study was conducted at Msambweni Hospital in Kwale County. Kwale County is located in the southern part of coastal Kenya. To the East is the Indian Ocean beach and borders Tanzania from the south and south western regions. It forms an important portal of entry and exit from Tanzania and the larger southern Africa region. Describe the population dynamics to give a picture of numbers of children. According to the department of vaccines and immunisation of Kenya, as by 25 th September 2012, Kwale County had 28 suspected measles cases of which 24 were laboratory confirmed (IgM positive). ${ }^{9,29}$ The county benefited from a measles immunisation programme supplemented with vitamin $\mathrm{A}$ given to children aged between 9 and 59 months. This Campaign was conducted between 3rd and 7th November 2012 and covered approximately $73.9 \%$ of the target population. ${ }^{12}$

Lamu County: Part of the study participants were drawn from King Fahd Hospital in Lamu County. Lamu County is located in the Northern coast of Kenya bordering Indian Ocean to the East, Somalia to the North East and Kilifi County to the South. During the 2011/2012 measles outbreaks Lamu County was one of the five counties in Kenya not to record a confirmed measles case, and the only county to record a single suspected case, the lowest in Kenya. ${ }^{9,10}$ Nevertheless, the county benefited from a measles mop-up immunisation programme supplemented with vitamin A given to children aged between 9 and 59 months. This Campaign was conducted between 3rd and 7th November 2012 and covered approximately $92.0 \%$ of the target population. $^{12}$

Narok County: A part of the study participants were drawn from Narok County. A region found within the Great Rift Valley and is made up of 3 constituencies; Narok North, Kilgoris and Narok South. As by September 2012, Narok county had 441 suspected measles cases of which 45 were laboratory confirmed (IgM positive) and 324 patients were linked to positive cases with only 13 laboratory confirmed negative cases. ${ }^{9,10,29}$ According to the department of vaccines and immunisation in Kenya, the county benefited from supplemental measles immunisation programme (mop-up programme) with vitamin A given to children aged between 9 and 59 months. This Campaign was conducted between 17th and 21st September 2012 then repeated between 3rd and 7th November 2012 during the countrywide SIA campaign. ${ }^{12}$ Narok district Hospital serves mostly people from Narok North although it houses the county heads of the different health departments. Patients from Kilgoris and Narok South districts also do attend this hospital in considerable large numbers.

\section{Study population}

A total of 453 participants were randomly selected approximately on a ratio of (9:7:1) from Narok, Kwale and Lamu Counties respectively and according to the population of children below 5 years in the respective County as per 2009 census. Participants were recruited from selected hospitals from the 3 counties. The study targeted health looking children aged between 9 and 59 months attending the wellbeing (mother-child health) clinic and whose guardians were willing to enrol them into the study. Grossly ill participants and those whose guardians did not consent were left out. Guardians were given a brief explanation of the study and consented by a clinician (nurse in attendance).

\section{Demographic and risk factors information of participants}

An open ended questionnaire was used to collect information on participant's demographics, vaccination history and risk factors associated with measles outbreaks. Data was collected on children age, gender, history of suffering from measles, time when this occurred, vaccination status (any evidence), vaccination frequency, period since last vaccinated and residency. Guardian parameters were also collected including relationship with child, availability of child's vaccination card - antenatal care card (ANC), awareness about the Measles virus, awareness about Measles Vaccines and whether or not they experienced a measles outbreak, the approximate time and approximate number of people affected. This information was collected by a trained community health worker using a serialized standard demographic questionnaire.

\section{Sample collection, preparation and transportation}

Up to 200ul of blood was collected from finger or heel prick on to pre labelled standardized filter paper discs (Dry Blood Spot cards - DBS).The blood spots were allowed to air dry for 60 minutes before packaging. A natural formed free flowing drop is generally approximated to contain 0.05 millilitres of blood, our starting volume. ${ }^{30}$ Each dried filter paper was individually placed into a sealable plastic bag to prevent possible cross contamination and to protect from dust 
and moisture. The DBS cards were processed, packaged and then transported to the laboratory to await analysis at room temperature.

\section{Detection of Measles IgG antibody}

Dried blood was removed from the filter using a paper punch and placed together in $0.5 \mathrm{ml}$ of phosphate buffered saline (PBS). Specimen disks were then soaked for 30 minutes at ambient temperature. Filters were removed and any remaining liquid squeezed from them using duckbill forceps and added to the soaking liquid. Specimens processed from dried blood are considered to have a practical starting dilution of approximately 1:10. Samples were then analysed using ELISA kit from NOVATEC Immunodiagnostics GMBH (Nova Lisa ${ }^{\mathrm{TM}}$ ELISA) from Germany and optical density measured with a photometer set at $540 \mathrm{~nm}$. The results of the ELISA were calculated using the kit instructions and expressed in Nova Tec Units (NTU). Results were negative, positive or equivocal interpreted as not protected, protected or undeterminant respectively. All equivocal samples were retested; those that turned positive were considered as such while all those that turned negative or remained equivocal were regarded as negative for the purpose of our study.

\section{Detection HIV antibodies}

DBS samples were processed as above then were analysed using VironostikaR HIV Ag/Ab kit manufactured by BIOMERIEUX SA in Marcy-L' Etoile, France (Lot: A66BA, Ref: 259852). The optical density was measured at $450 \mathrm{~nm}$. All samples with absorbance of more than or equal to the cut-off absorbance (mean negative control $+0.100)$ were considered positive while samples with absorbance less than the cut-off were considered negative as per manufacturers advice. All reports were captured in a notebook initially, and then fed in to computer spread sheets, cleaned, verified for consistency and excel used to present it in form of charts and tables.

\section{Data Analysis and interpretation}

Statistical analysis was performed using SPSS version 20. Results from serological surveys were categorised as positive, negative or equivocal per participant. Data on demographics and risk factors was used to give counts, proportions and $95 \%$ confidence intervals (CI) for participants. The proportion seropositive was calculated for the general study population in relation to sex, age and risk factor groups with the exact $95 \% \mathrm{CI}$ of the proportions determined. The number and percentages of positive, negative and equivocal sera were calculated according to age in months, gender, and vaccination status and frequency. Our results were interpreted according to the manufacturer's recommendation as negative for titres below 9 NTUs, equivocal for titres between 9 and 11 NTUs, and positive for titres above 11 NTUs. Comparable to the 3rd international standards, 9 NTUs is equated to120 mill international units per millilitre (mIU/ $\mathrm{ml}$ ) while $11 \mathrm{NTUs}$ are equivalent to $220 \mathrm{mIU} / \mathrm{ml}$. Thus, negative titres were below $120 \mathrm{mIU} / \mathrm{ml}$, equivocal titres were between $120 \mathrm{mIU}$ and $220 \mathrm{mIU} / \mathrm{ml}$, and positive titres were those found to be above $220 \mathrm{mIU} /$ $\mathrm{ml}$ which is slightly above the widely accepted measles protective titre of $200 \mathrm{mIU} / \mathrm{ml}^{31-33}$ Seroprotectivity was compared with risk factors and associations drawn in terms of odds ratios (OR) and likelyhoodness using chi-square and student $t$ tests. The data from this work was presented in tables, histograms and charts. A p-value of less than 0.05 was considered statistically significant.

\section{Results and Discussion}

\section{Baseline characteristics of study participants}

A total of 453 children were studied. Out of these, $210(45.85 \%)$ were from Narok, 185 (41.27\%) from Kwale and 58 (12.88\%) from Lamu Counties respectively. Out of 453, $223(48.68 \%)$ were female, while $235(51.31 \%)$ were male. $103(46.18 \%)$ of females were from Narok, 93 (41.7\%) from Kwale, while 27 (12.11\%) were from Lamu. 107 of $235(45.53 \%)$ men were from Narok, $96(40.85 \%)$ from Kwale and $32(13.62 \%)$ from Lamu. In Narok 103 out of $210(49.05 \%)$ were female and 107 out of $210(50.95 \%)$ were male, Kwale 93/185 (49.21\%) female and 96/185 (50.79\%) male and Lamu 27/58 (45.76\%) female and 32/58 (54.24\%) were male (Tables 1-3), (Figure 1).

Table I Baseline characteristics of study participants: Socio-demographics and associated risk factor attributes

\begin{tabular}{|c|c|c|c|c|c|c|c|c|c|c|}
\hline \multirow{2}{*}{ Category } & \multicolumn{2}{|c|}{ Kwale } & \multicolumn{2}{|c|}{ Lamu } & \multicolumn{2}{|c|}{ Narok } & \multicolumn{2}{|c|}{ Totals } & \multicolumn{2}{|c|}{ 95\% Cl Limits } \\
\hline & $\mathbf{N}$ & $\%$ & $\mathbf{N}$ & $\%$ & $\mathbf{N}$ & $\%$ & $\mathbf{N}$ & $\%$ & Lower & Upper \\
\hline \multicolumn{11}{|l|}{ Sex } \\
\hline Male & 94 & 50.8 & 32 & 55.2 & 107 & 51 & 233 & 51.4 & 46.7 & 56.1 \\
\hline Female & 91 & 49.2 & 26 & 44.8 & 103 & 49 & 220 & 48.6 & 44.1 & 53 \\
\hline \multicolumn{11}{|l|}{ Age Group } \\
\hline$\leq 12$ & 27 & 14.6 & 3 & 5.2 & 14 & 6.7 & 44 & 9.7 & 8.9 & 10.6 \\
\hline $12 \_24$ & 65 & 35.1 & 12 & 20.7 & 60 & 28.6 & 137 & 30.2 & 27.5 & 33 \\
\hline $24-36$ & 46 & 24.9 & 12 & 20.7 & 71 & 33.8 & 129 & 28.5 & 25.9 & 31.1 \\
\hline $36-48$ & 26 & 14.1 & 15 & 25.9 & 48 & 22.9 & 89 & 19.6 & 17.9 & 21.4 \\
\hline $48-60$ & 21 & 11.4 & 16 & 27.6 & 17 & 8.1 & 54 & 11.9 & 10.9 & 13 \\
\hline \multicolumn{11}{|l|}{ Guardian } \\
\hline Parent & 174 & 94.1 & 36 & 62.1 & 189 & 90 & 399 & 88.1 & 80 & 96.1 \\
\hline Brother/Sister & 4 & 2.2 & 2 & 3.5 & 18 & 8.6 & 24 & 5.3 & 4.9 & 5.7 \\
\hline Uncle/Aunty & 4 & 2.2 & 1 & 1.7 & 2 & 1 & 7 & 1.6 & 1.5 & 1.6 \\
\hline Cousin/Friend & 3 & 1.6 & 19 & 32.8 & I & 0.5 & 23 & 5.1 & 4.7 & 5.5 \\
\hline \multicolumn{11}{|c|}{ Awareness about $M$ vaccines } \\
\hline Yes & 173 & 93.5 & 56 & 96.6 & 184 & 87.6 & 413 & 91.2 & 82.8 & 99.6 \\
\hline No & 12 & 6.5 & 2 & 3.4 & 26 & 12.4 & 40 & 8.8 & 8 & 9.6 \\
\hline \multicolumn{11}{|c|}{ Source of Information } \\
\hline Health worker & 105 & 60.7 & 46 & 82.1 & 129 & 70.1 & 280 & 67.8 & 61.3 & 74.3 \\
\hline Others & 68 & 39.3 & 10 & 17.9 & 55 & 29.9 & 133 & 32.2 & 29.1 & 35.3 \\
\hline \multicolumn{11}{|c|}{ Vaccination Card } \\
\hline Yes & 152 & 82.2 & 45 & 77.6 & 149 & 71 & 346 & 76.4 & 69.4 & 83.4 \\
\hline
\end{tabular}


Table Continued..

\begin{tabular}{|c|c|c|c|c|c|c|c|c|c|c|}
\hline \multirow{2}{*}{ Category } & \multicolumn{2}{|c|}{ Kwale } & \multicolumn{2}{|c|}{ Lamu } & \multicolumn{2}{|c|}{ Narok } & \multicolumn{2}{|c|}{ Totals } & \multicolumn{2}{|c|}{ 95\% Cl Limits } \\
\hline & $\mathbf{N}$ & $\%$ & $\mathbf{N}$ & $\%$ & $\mathbf{N}$ & $\%$ & $\mathbf{N}$ & $\%$ & Lower & Upper \\
\hline No & 33 & 17.7 & 13 & 22.4 & 61 & 29.1 & 107 & 23.6 & 21.5 & 25.7 \\
\hline \multicolumn{11}{|c|}{ Vaccination Status } \\
\hline Vaccinated & 178 & 96.2 & 55 & 94.8 & 175 & 83.3 & 408 & 90.1 & 81.8 & 98.4 \\
\hline Not & 3 & 1.6 & 2 & 3.4 & 6 & 2.9 & II & 2.4 & 2.2 & 2.6 \\
\hline Don't know & 4 & 2.2 & I & 1.7 & 29 & 13.8 & 34 & 7.5 & 6.9 & 8.1 \\
\hline \multicolumn{11}{|c|}{ Vaccination Times } \\
\hline Once & 167 & 90.2 & 36 & 62.1 & $15 \mid$ & 71.9 & 354 & 86.8 & 78.4 & 95.1 \\
\hline Twice & 11 & 6 & 14 & 24.1 & 24 & $1 \mathrm{I} .4$ & 49 & 12 & 10.9 & 13.1 \\
\hline Thrice & 0 & 0 & 5 & 5.2 & 0 & 0 & 5 & 1.2 & 1.2 & $\mathrm{I} .3$ \\
\hline \multicolumn{11}{|c|}{ HIV Seropositivity } \\
\hline Positive & 13 & 7 & 5 & 8.6 & 13 & 6.2 & 31 & 6.8 & 6.3 & 7.4 \\
\hline Negative & 172 & 93 & 53 & 91.4 & 197 & 93.8 & 422 & 93.2 & 84.6 & 101.7 \\
\hline
\end{tabular}

Table 2 Measles sero status by sociodemographic and risk factors

\begin{tabular}{|c|c|c|c|c|}
\hline \multicolumn{5}{|c|}{ Measles IgG Seroprotectivity Per Risk Group } \\
\hline \multirow{2}{*}{ Category } & \multirow{2}{*}{ Total } & Protected & Not Protected & Borderline \\
\hline & & $n(\%)$ & $n(\%)$ & n (\%) \\
\hline Overall & 453 & $376(83.0)$ & $57(12.6)$ & 20(4.4) \\
\hline \multicolumn{5}{|l|}{ Gender } \\
\hline Male & 233 & $197(84.6)$ & $28(12.0)$ & $8(3.4)$ \\
\hline Female & 220 & 179(81.4) & $29(\mid 3.2)$ & $12(5.5)$ \\
\hline \multicolumn{5}{|l|}{ Age in Months } \\
\hline Below 12 & 44 & $33(75.0)$ & $8(18.2)$ & $3(6.8)$ \\
\hline $12-24$ & 137 & $118(86.1)$ & $14(10.2)$ & $5(3.7)$ \\
\hline $24-36$ & 129 & $108(83.7)$ & $16(12.4)$ & $5(3.9)$ \\
\hline $36-48$ & 89 & $75(84.3)$ & $\mathrm{II}(\mathrm{I} 2.4)$ & $3(3.4)$ \\
\hline $48-60$ & 54 & $44(8 I .5)$ & $8(14.8)$ & $2(3.7)$ \\
\hline \multicolumn{5}{|l|}{ County } \\
\hline Kwale & 185 & I58(85.4) & $18(9.7)$ & $9(4.9)$ \\
\hline Narok & 210 & $168(80.0)$ & $32(15.2)$ & $10(4.8)$ \\
\hline Lamu & 58 & $50(86.2)$ & $7(12.1)$ & $\mathrm{I}(\mathrm{I} .7)$ \\
\hline \multicolumn{5}{|c|}{ Vaccination Status } \\
\hline Vaccinated & 408 & $346(84.8)$ & $46(I 1.3)$ & $16(3.9)$ \\
\hline Not vaccinated & II & $2(18.2 \%)$ & $6(54.5)$ & $3(27.3)$ \\
\hline Don't know & 34 & $28(82.4)$ & $5(14.7)$ & $\mathrm{I}(2.9)$ \\
\hline \multicolumn{5}{|c|}{ Vaccination Frequency } \\
\hline Ones & 354 & 298(84.2) & $4 I(I I .6)$ & $15(4.2)$ \\
\hline Twice & 49 & $43(87.8)$ & $5(10.2)$ & $\mathrm{I}(2.0)^{\prime}$ \\
\hline Thrice & 5 & $5(100)$ & 0 & 0 \\
\hline \multicolumn{5}{|c|}{ Awareness about Vaccines } \\
\hline Aware & 413 & $344(83.3)$ & $50(12.1)$ & $19(4.6)$ \\
\hline Not aware & 40 & $32(80.0)$ & $7(17.5)$ & $\mathrm{I}(2.5)$ \\
\hline \multicolumn{5}{|c|}{ HIV Seropositivity } \\
\hline Seropositive & 31 & $5(16.1)$ & $24(77.4)$ & $2(6.5)$ \\
\hline Seronegative & 422 & $37 I(87.9)$ & $33(7.8)$ & $18(4.3)$ \\
\hline
\end{tabular}

Table 3 Measles Seroprotectivity in relation to associated risk factors

\begin{tabular}{|c|c|c|c|c|c|c|c|c|}
\hline \multirow{2}{*}{ Factor } & \multirow{2}{*}{ Examined (n) } & \multirow{2}{*}{ Protected (n) } & \multirow{2}{*}{ Prevalence (\%) } & \multirow{2}{*}{ Chi Square } & \multirow{2}{*}{$P$ value } & \multirow{2}{*}{ Odds Ratio } & \multicolumn{2}{|c|}{ 95\% Cl limits } \\
\hline & & & & & & & Lower & Upper \\
\hline \multicolumn{9}{|l|}{ Gender } \\
\hline Female & 220 & 179 & 81.4 & \multirow{2}{*}{0.814} & \multirow{2}{*}{0.367} & \multirow{2}{*}{1.253} & \multirow{2}{*}{0.767} & \multirow{2}{*}{2.049} \\
\hline Male & 233 & 197 & 84.6 & & & & & \\
\hline \multicolumn{9}{|c|}{ Vaccination Card } \\
\hline No & 107 & 68 & 63.6 & \multirow[t]{2}{*}{37.566} & \multirow{2}{*}{0} & \multirow{2}{*}{4.649} & \multirow{2}{*}{2.769} & \multirow{2}{*}{7.805} \\
\hline Yes & 346 & 308 & 89 & & & & & \\
\hline \multicolumn{9}{|c|}{ Awareness about Vaccines } \\
\hline Not aware & 40 & 32 & 80 & \multirow{2}{*}{0.28} & \multirow{2}{*}{0.597} & \multirow{2}{*}{1.246} & \multirow{2}{*}{0.551} & \multirow{2}{*}{2.821} \\
\hline Aware & 413 & 344 & 83.3 & & & & & \\
\hline
\end{tabular}


Table Continued...

\begin{tabular}{|c|c|c|c|c|c|c|c|c|}
\hline \multirow{2}{*}{ Factor } & \multirow{2}{*}{ Examined (n) } & \multirow{2}{*}{ Protected (n) } & \multirow{2}{*}{ Prevalence (\%) } & \multirow{2}{*}{ Chi Square } & \multirow{2}{*}{$P$ value } & \multirow{2}{*}{ Odds Ratio } & \multicolumn{2}{|c|}{ 95\% CI limits } \\
\hline & & & & & & & Lower & Upper \\
\hline \multicolumn{9}{|c|}{ Source of Information } \\
\hline Health workers & 280 & 231 & 82.5 & \multirow{2}{*}{0.393} & \multirow{2}{*}{0.531} & \multirow{2}{*}{1.198} & \multirow{2}{*}{0.68} & \multirow{2}{*}{2.112} \\
\hline Others & 133 & 113 & 85 & & & & & \\
\hline \multicolumn{9}{|c|}{ Vaccination Status } \\
\hline Yes & 408 & 346 & 84.8 & \multirow{2}{*}{33.78} & \multirow{2}{*}{0} & \multirow{2}{*}{0.04} & \multirow{2}{*}{0.008} & \multirow{2}{*}{0.189} \\
\hline No & 11 & 2 & 18.2 & & & & & \\
\hline \multicolumn{9}{|c|}{ Vaccination Frequency } \\
\hline Once & 354 & 298 & 84.2 & \multirow[t]{2}{*}{0.806} & \multirow{2}{*}{0.369} & \multirow{2}{*}{1.503} & \multirow{2}{*}{0.614} & \multirow{2}{*}{3.681} \\
\hline Twice & 54 & 48 & 88.9 & & & & & \\
\hline \multicolumn{9}{|l|}{ HIV Status } \\
\hline Negative & 422 & 371 & 87.9 & \multirow{2}{*}{105.48} & \multirow{2}{*}{0} & \multirow{2}{*}{0.026} & \multirow{2}{*}{0.1} & \multirow{2}{*}{0.72} \\
\hline Positive & 31 & 5 & 16.1 & & & & & \\
\hline
\end{tabular}

\section{Frequency and Proportions of Vaccination}

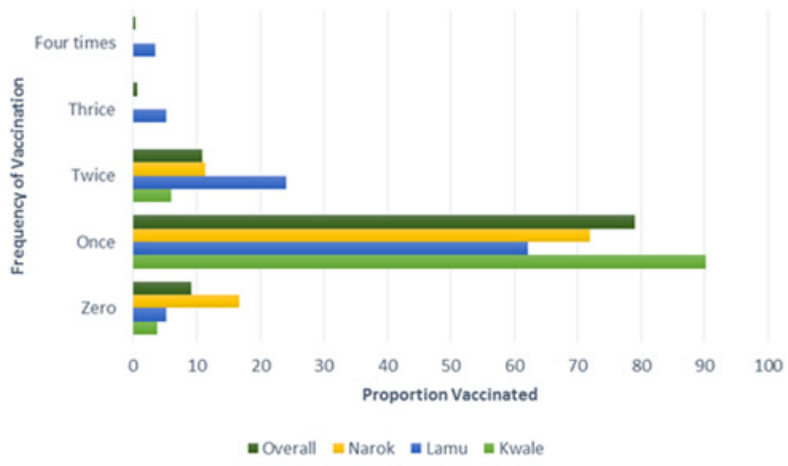

Figure I Bar chart showing proportion vaccinated for each frequency category in the three Counties.

\section{Discussion}

This study brings open, a rare glimpse, at least for the first time in to the sero epidemiological profile of Measles-specific IgG antibodies among a sub population of Kenyan children. Our results suggest that the general population sero - immunity among children between 9 to 59 months as in 2014 , was $83.0 \%$, well below the acceptable herd immunity threshold (93-95\%) necessary to interrupt measles transmission as suggested by several studies. ${ }^{14,19,32}$ Comparatively, this is much higher than what another recent (2011) study in Bangui, Central African Republic (CAR) found $(57.3 \%$ ) on a research conducted in 2008 despite having reached immunization coverage's of more than $90 \%$ in $2006 .{ }^{34}$ Whereas CAR is among the regions poorest countries with Human Development Index (HDI) of 0.352, Kenya is among the medium developing countries, HDI of $0.555 . .^{35}$ And this could probably be the attributing factor for the difference in sero-immunity despite having almost the same immunization coverage's albeit at slightly different times. Among the vaccinated, protection stood at $84.8 \%$ whereas among the non-vaccinated protection was at $18.2 \%$. This suggests that vaccination using the $\mathrm{McV}$ is very effective as far as protection against measles is concerned considering the significant association between vaccination and protection (Chi-square $=33.672$; $\mathrm{P}<0.001$ ). Such association has been emphasized by world health organization. ${ }^{19,36,37}$ And mentioned in reports from other studies as well..$^{14,20,38}$ The odds of being seronegative were higher for residents of Narok County as compared to Kwale or Lamu counties. This could have been attributed to factors such as frequent movements of people, land Scape, and distance from health centers which probably limit access to immunization services or population targeted awareness programmes. This is complimented by the fact that this county had the least proportion of vaccinated individuals when compared to the other two.

Although there was no significant difference in protection between male and female populations $(Z=0.9022, P=0.36812)$, the risk of not being protected was higher in females than males (Odds ratio $=$ $1.253,95 \%$ CI; $0.767-2.069$ ). There was no significant difference in seroprotectivity between children whose guardians were aware of Measles containing vaccines $(\mathrm{McV})$ and those not aware (Chi-square $=0.28, \mathrm{P}=0.597)$. Despite there being no significant difference, $\mathrm{P}>$ 0.05 , the risk of not being protected was higher among children whose parents were not aware about the measles containing vaccine $(\mathrm{McV})$ as compared to children whose parents were aware about the vaccine $(80.0 \%$ vs $83.3 \%$; Odds ratio $=1.246 ; 95 \%$ CI, $0.551-2.821)$. Moreover, children whose guardians had no vaccination cards were less likely to be protected with the specific measles IgG antibodies than those with vaccination cards $(61.7 \%$ vs $89.6 \%$; Odds ratio $=$ $5.349 ; 95 \%$ CI, 3.178 - 9.003), and, there was a significant difference in seroprotectivity between these two groups (Chi-square $=45.133 ; \mathrm{P}$ $<0.001)$. Incidentally, such comparative studies are extremely scanty; we couldn't get research work to compare with our study. Although there is scant data on awareness studies, awareness of $\mathrm{McV}$ seems to plays a major role on decision making when it comes to availing children for vaccinations. This idea is brought afore by the existence of a significant difference in proportion of guardians (Chi-square $=$ $20.283, \mathrm{P}<0.0001)$, aware about $\mathrm{McV}(79.2 \%)$ compared to those not aware about $\mathrm{McV}(47.5 \%)$, when it comes to carrying of vaccination cards. Consecutively, there was also a significant difference (Chisquare $=51.748, \mathrm{P}<0.0001)$ between these two groups in relation to vaccination status. In guardians aware about the $\mathrm{McV}, 93.2 \%$ were vaccinated compared to only $57.5 \%$ in those guardians not aware about the McV. This shows that awareness of McV plays a role on the overall population immunity in the study group. Among children aged between 9 and 59 months, vaccinated children had more chances of having measles-specific $\operatorname{IgG}$ protective antibodies than those that were unvaccinated and this was significantly so at $95 \%$ CI $(84.5 \%$ vs. $18.2 \%$; chi-square $=33.671 ; \mathrm{P}<0.0001)$. $\mathrm{McV}$ are an integral part in protection against measles, this has been proved beyond doubt in several studies. ${ }^{8,20,39}$ And hence are next only to Measles natural infection as far as protection against measles disease is concerned. In another study, $84.8 \%$ of confirmed measles cases were found not to have been vaccinated. ${ }^{40}$ A greater number of HIV seronegative children had measles specific IgG protective antibodies compared to HIV seropositivity children, the difference is statistically significant (Chi-square $=105.48, \mathrm{P}<0.0001)$. The risk of not being protected was lower in HIV seronegative children than HIV sero positive children 
$(16.1 \%$ Vs $87.9 \%$; Odds ratio $=0.026 ; 95 \%$ CI, 0, 01-0.072). These results concur well with other studies done elsewhere which showed the existence of a measles immunity gap between HIV positive and HIV negative children..$^{34,41,42}$ Low measles seroprotectivity among HIV sero positive children may be attributed to impaired vaccination response due to compromised immune system at the time of vaccination or accelerated waning of immunity due to impaired immunity. ${ }^{19,34,41,43}$

As per the relationship between natural infection and vaccination, in this study, we found no significant difference in association between immunity attained through vaccination with the measles containing vaccine or through natural infection with the wild measles virus $(83.9 \%$ vs. $84.6 \%$; chi-square $=0.005 ; p=0.9478)$. However, although there was no significant difference in protection, it was shown that vaccinated children who also suffered from Measles infection had more chances of being sero protected than those who didn't suffer from the disease $(84.6 \%$ vs $83.9 \%$; Odds ratio $=1.0525$ $95 \% \mathrm{CI}=0.2277$ to $4.862 ; \mathrm{P}=0.9478)$. Natural immunity has always been proven to be superior to vaccine induced immunity and our case is not an exception. ${ }^{37,44}$ Generally, a titer of more than $200 \mathrm{mIU} /$ $\mathrm{ml}$ is considered protective as suggested elsewhere. ${ }^{33,45}$ Although, re infection and disease may not be completely ruled out in individuals whose immune systems have had previous priming through the wild or vaccine strains. ${ }^{31,45,46}$ Attaining of protective titers notwithstanding. In our study however, we set the estimated "protective" threshold at $220 \mathrm{mIU} / \mathrm{ml}$ on the basis of the manufacturer advised optical density measurements.

There was a significant difference between the measles IgG seropositivity rate in the region of study when compared against reported vaccination intake $(83.0 \%$ vs $90.1 \% ; \mathrm{Z}=3.1144, \mathrm{P}=$ 0.00188 ). Reduced vaccine efficacy could have arisen due to compromised vaccine, poor host characteristics that adversely affected seroconversion rates. This, coupled with the now greatly reduced global exposure rates to circulating wild measles virus may play a significant role. Potential confounding limitations of our study; Subject selection was based on hospital/clinic attendance which could have biased our results, and, levels of protective antibodies may not have been fully and rightly categorized because of the method applied and the diagnostic kit used.

\section{Conclusion}

The sero epidemiological study revealed that a huge number of children did not have protective antibody titers against measles despite the wide vaccine coverage. Vaccination awareness was low among the adult study population and large number of children lacked vaccination records.

\section{Recommendations}

Measures need to be put in place to monitor efficacy of vaccination programmes, such measures may include automated vaccination records for all children, awareness on importance of vaccination cards and attendance of child health clinics, yearly large-scale serological surveillance. In the meantime, the quality of vaccination activities (routine and supplementary immunization campaigns) must be improved, and population awareness of their importance must be increased. Monitoring routine immunization coverage to assess administrative coverage levels, which are habitually unreliable, and robust methods to monitor and evaluate supplementary immunization activities are needed.

\section{Acknowledgements}

The author acknowledges the support of staff from the medical sciences departments of Jomo Kenyatta University and the Technical University of Mombasa. We also acknowledge the technical involvement of Mr. Paul Muchiri, and Mr Chegge from centre for virus research, Kenya Medical Research Institute, and, staff from Narok district Hospital, King Fahd Hospital from Lamu and Msambweni Referral Hospital from Kwale County. Author acknowledges funding from the National Commission for Science Technology and Innovation (NACOSTI) of Kenya which partially facilitated the research.

\section{Conflicts of Interest}

None expressed.

\section{References}

1. (2011) Centers for Disease Control and Prevention. Measles -Horn of Africa, 2010-2011. Morb Mortal Wkly report 61(34): 678-684.

2. Perry RT, Gacic-Dobo M, Dabbagh A, Mulders MN, Strebel PM, et al. (2014) Global control and regional elimination of measles, 2000-2012. MMWR Morb Mortal Wkly Rep 63(5): 103-107.

3. World Health Organization (2013) Progress in global control and regional elimination of measles, 2000-2011. Wkly Epidemiol Rec 3(88): 29-36

4. Simons E, Mort M, Dabbagh A, Strebel P, Wolfson L (2011) Strategic planning for measles control: Using data to inform optimal vaccination strategies. J Infect Dis 204 (Suppl 1): 28-34.

5. Naim HY (2015) Measles virus. Hum Vaccin Immunother 11(1):21-6

6. (1993) Sigerist HE the Great doctors, Dover Publications, WW Norlon and Company, Inc, USA, pp.78-87.

7. Holmlund H (2012) Rhazes: A Pioneer in Clinical Observation, USA, p 20.

8. Antona D, Lévy-Bruhl D, Baudon C, Freymuth F, Lamy M, et al. (2013) Measles elimination efforts and 2008-2011 outbreak, France. Emerg Infect Dis 19(3):357-364.

9. World Health Organization (2012) Measles outbreak in Kenya. Kenya Health sector Bulletin 2-3.

10. (2011) Centers for Disease Control and Prevention. Measles -Horn of Africa, 2010-2011. Morb Mortal Wkly report 61(34): 678-684.

11. World Health Organization (2011) Centers For Disease Control And Prevention Cdc. Measles outbreaks and progress toward measles preelimination, African region, 2009-2010. Mmwr Morb Mortal Wkly Rep 60 (12): 374-378.

12. Steegen K, Luchters S, Dauwe K, Reynaerts J, Mandaliya K, et al. (2009) Effectiveness of antiretroviral therapy and development of drug resistance in HIV-1 infected patients in Mombasa, Kenya. AIDS Res Ther 6: 12 .

13. IRIN (2012) Major measles vaccine campaign planned in Kenya.

14. Penedos AR, Myers R, Hadef B, Aladin F, Brown KE (2015) Assessment of the utility of whole genome sequencing of measles virus in the characterisation of outbreaks. PLoS One 10(11): 1-16.

15. Fulton BO, Sachs D, Beaty SM, Won ST, Lee B, et al. Mutational Analysis of Measles Virus Suggests Constraints on Antigenic Variation of the glycoproteins. Cell Rep 11(9): 1331-1338.

16. Gillet Y, Steri GC, Behre U, Arsène JP, Lanse X, et al. (2009) Immunogenicity and safety of measles-mumps-rubella-varicella (MMRV) vaccine followed by one dose of varicella vaccine in children aged 15 months-2 years or 2-6 years primed with measles-mumpsrubella (MMR) vaccine. Vaccine 27(3): 446-453. 
17. Tahara M, Ito Y, Brindley MA, Ma X, He J, et al. (2013) Functional and Structural Characterization of Neutralizing Epitopes of Measles Virus Hemaggltinin Protein. J Virol 87(1): 666-675.

18. Chandwani S, Beeler J, Li H, Audet S, Smith B, et al. (2011) Safety and immunogenicity of early measles vaccination in children born to HIV-infected mothers in the United States: Results of Pediatric AIDS Clinical Trials Group (PACTG) protocol 225. J Infect Dis 204(Suppl 1): 179-189.

19. World Health Organization (2009) Measles vaccines: WHO position paper - 28 August 2009 Grading of scientific evidence in support of key recommendations, $86-7$.

20. Uzicanin A, Zimmerman L (2011) Field effectiveness of live attenuated measles-containing vaccines: A review of published literature. J Infect Dis 204(Suppl 1): S133-148.

21. Bankamp B, Takeda M, Zhang Y, Xu W, Rota PA (2011) Genetic Characterization of Measles Vaccine Strains. J Infect Dis 204(Suppl1) S533-S548.

22. Rodrigues A, Ravn H, Whittle HC (2008) Protective efficacy of standard Edmonston-Zagreb measles, BMJ.

23. Martins C, Garly ML, Bale C, Rodrigues A, Benn CS, Whittle H, et al. (2013) Measles antibody levels after vaccination with EdmonstonZagreb and Schwarz measles vaccine at 9 months or at 9 and 18 months of age : A serological study within a randomised trial of different measles vaccines. Vaccine 31(48): 5766-5771.

24. Centers for Disease Control and Prevention (CDC) (2013) Progress in increasing breastfeeding and reducing racial/ethnic differences - United States, 2000-2008 births. MMWR Morb Mortal Wkly Rep 62(5): 77-80.

25. Li J, Lu L, Pang X, Sun M, Ma R, et al. (2013) A 60-year review on the changing epidemiology of measles in capital Beijing, China, 1951-2011. BMC Public Health 13: 1-986.

26. Durrheim DN, Crowcroft NS, Strebel PM (2014) Measles- The epidemiology of elimination. Vaccine 32(51): 6880-6883.

27. Subaiya S, Dumolard L, Lydon P, Gacic-dobo M, Eggers R, et al. (2015) Global routine vaccination coverage, 2014. WHO Wkly Epidemiol Rec 64(44): 1252-1255.

28. Perry RT, Gacic-Dobo M, Dabbagh A, Strebel P, Okwo-Bele JM, et al. (2014) Global control and regional elimination of measles, 2000-2011. MMWR 63(5): 103-107.

29. (2012) DVI Measles Brief-1.

30. Bond MM, Richards-Kortum RR (2015) Drop-to-drop variation in the cellular components of fingerprick blood: Implications for point-of-care diagnostic development. Am J Clin Pathol 144(6): 885-894.

31. Manirakiza A, Kipela JM, Sosler S, Daba RM, Gouandjika-Vasilache I (2011) Seroprevalence of measles and natural rubella antibodies among children in Bangui, Central African Republic. BMC Public Health. BMC Public Health 11(1): 1-327.

32. Poethko-Müller C, Mankertz A (2012) Seroprevalence of measles-, mumps- and rubella-specific IgG antibodies in German children and adolescents and predictors for seronegativity. PLoS One 7(8): e42867.
33. Kizito D, Tweyongyere R, Namatovu A, Webb EL, Muhangi L, et al. (2013) Factors affecting the infant antibody response to measles immunisation in Entebbe-Uganda. BMC Public Health 619(13): 1-9.

34. Manirakiza A, Kipela JM, Sosler S, Daba RM, Gouandjika-Vasilache I (2011) Seroprevalence of measles and natural rubella antibodies among children in Bangui, Central African Republic. BMC Public Health 11(1): 327.

35. UNDP Human Development Report 2012 Africa Human Development Report 2012. Towards a Food Secure Future 1-190.

36. Perry RT, Gacic-dobo M, Dabbagh A, Mulders MN, Strebel PM, et al. (2014) Progress Toward Regional Measles Elimination-Worldwide, 2000-2014. Morbidity and Mortality Weekly Report (MMWR) 63(45): 1246-1251.

37. Organization WHO (2017) Weekly epidemiological record: relevé épidémiologique hebdomadaire. Wkly Epidemiol Rec 21(92): 293-300.

38. Strebel PM, Cochi SL, Hoekstra E, Rota PA, Featherstone D, et al. (2011) A world without measles. J Infect Di 204(SUPPL 1): 1-3.

39. Fannie Defay, Gaston De Serres, Danuta M. Skowronski, Nicole Boulianne, et al. (2013) Measles in children vaccinated with 2 doses of MMR. Pediatrics 132(5): 1-10.

40. (2016) European Centre for Disease Prevention and Control. Measles and rubella monitoring, January 2016-Reporting on January 2015-December 2015 Surveillance data and epidemic inteligence data until 31 January 2016. Stockholm.

41. Kizito D, Tweyongyere R, Namatovu A, Webb EL, Muhangi L, et al. (2013) Factors affecting the infant antibody response to measles immunisation in Entebbe-Uganda. BMC Public Health 13(1): 619.

42. Siberry GK, Patel K, Bellini WJ, Karalius B, Purswani MU, et al. (2015) Immunity to Measles, Mumps, and Rubella in US Children with Perinatal HIV Infection or Perinatal HIV Exposure Without Infection. Clin Infect Dis 61(6): 988-995.

43. Durando P, Esposito S, Bona G, Cuccia M, Giuseppina M, et al. (2017) The immunogenicity and safety of a tetravalent measles-mumps- rubellavaricella vaccine when co-administered with conjugated meningococcal $\mathrm{C}$ vaccine to healthy children : A phase IIIb, randomized, multi-center study in Italy q. Vaccine Kidlington 34(36): 4278-484.

44. Olaitan AE, Ella EE, Ameh JB (2015) Comparative seroprevalence of measles virus immunoglobulin $\mathrm{M}$ antibodies in children aged 0-8 months and a control population aged 9-23 months presenting with measles-like symptoms in selected hospitals in Kaduna State. Int J Gen Med 8: 8-101.

45. Chen RT, Markowitz LE, Albrecht P, Stewart JA, Lynne M, et al. (1990) Measles Antibody: Reevaluation of Protective Titers. J Infect Dis 162(5): 1036-1042.

46. Fu C, Xu J, Liu W, Zhang W, Wang M, et al. (2010) Low measles seropositivity rate among children and young adults: a seroepidemiological study in southern China in 2008. Vaccine 28(51): 82198223. 